\title{
Optic Neuritis After a Snakebite: A Diagnostic Dilemma
}

\author{
Jeyhan Dhabhar, MD, ${ }^{1}$ Varshil Mehta, MBBS, ${ }^{2}$ Nimit Desai, MD $^{3}$ \\ ${ }^{1}$ Department of Internal Medicine, MGM Medical College, Navi Mumbai, India ²Department of Cardiology, Lister Hospital, Stevenage, UK \\ ${ }^{3}$ Department of Neurology, Medical University of South Carolina, Charleston, SC, USA
}

Background: Snakebite is one of the major causes of morbidity and mortality in India, particularly in rural regions. Of the 57 known venomous species of snakes in India, the 4 most dangerous snakes are the cobra, the common krait, the Russell viper, and the sawscaled viper. Of these, the snakes commonly implicated with neurotoxicity are the cobra and the common krait-both elapidae. Acute neuromuscular weakness with respiratory system involvement is the most lethal neurotoxic effect.

Case Report: A 24-year-old female was brought to the emergency department in an unresponsive state with a history of snakebite on the left foot. The patient was intubated, mechanically ventilated, and promptly started on snake antivenom and anticholinesterase agents. The patient improved significantly and was extubated. On day 6 , she developed blurred vision and slurred speech. She was diagnosed with bilateral optic neuropathy and treated with repeat snake antivenom and steroids. She improved significantly and was discharged on day 14.

Conclusion: When a person is bitten by a venomous snake, antivenom is the mainstay of treatment, but clinicians must also consider possible reactions and complications. Optic neuritis following a snakebite is rare but does occur. The prognosis is generally good if clinical suspicion for such a complication is strong, the snake is identified, and the patient receives timely treatment with steroids.

Keywords: Delayed diagnosis, elapidae, neurotoxicity syndromes, snake bites

Address correspondence to Varshil Mehta, MBBS, Department of Cardiology, Lister Hospital, Coreys Mill Ln., Stevenage SG1 4AB, UK. Tel: +44730 747 4454. Email: varshil.mehta@nhs.net

\section{INTRODUCTION}

In India, a developing country where the principal occupation is agriculture, snakes and other reptiles form an important part of the ecosystem. The World Health Organization (WHO) has classified snakebite as a neglected tropical disease of global importance. ${ }^{1}$ Kasturiratne et al estimated that at least 1.2 million snakebites occur annually worldwide, with approximately 421,000 envenomings and 20,000 deaths. ${ }^{2}$ In India, the estimated number of snakebites is higher than reported figures, as many victims choose traditional therapies and most die outside of hospitals. ${ }^{3}$

India has more than 242 snake species, 57 of which are highly poisonous and venomous. The 4 most dangerous species are the Indian cobra (Naja naja), the common krait (Bungarus caeruleus), the Russell viper (Daboia russelii), and the saw-scaled viper (Echis carinatus). ${ }^{4}$

A well-known result of envenoming by kraits (Bungarus spp) and cobras (Naja spp) is neurotoxicity. Acute neuromuscular paralysis is the primary presentation of neurotoxicity and is a major cause of morbidity and mortality related to snakebites. However, timely administration of snake antivenom and ventilatory assistance can help prevent mortality and morbidity. ${ }^{4}$ We describe the case of a 24year-old female who presented with snakebite from an Indian cobra.

\section{CASE REPORT}

A 24-year-old female was brought to the emergency room in an unresponsive and gasping state. She had been bitten by a snake on the dorsal aspect of her left foot approximately 1 hour before presentation. The patient sustained the snakebite when she was walking in a garden. Approximately 15 to 20 minutes following the bite, she developed bilateral severe ptosis followed by dyspnea that was rapidly progressive, explaining why the patient was gasping for breath when she reached the hospital. She had no significant medical history or comorbidities. The patient's relatives brought the snake to the hospital, and it was identified as an Indian spectacled cobra (Figure, left panel).

The patient's oxygen saturation $\left(\mathrm{SpO}_{2}\right)$ was $70 \%$ to $80 \%$ on high-flow oxygen. Other vitals were stable. Physical examination was unremarkable except for the bite marks on the left foot. Peripheral pulses were felt in all 4 limbs. Because of the patient's respiratory distress, she was intubated and provided with ventilatory support.

Whole blood clotting test was normal. The patient was started on polyvalent snake antivenom (snake venom antiserum IP [Indian pharmacopeia], lyophilized) 10 vials diluted in $500 \mathrm{~mL}$ of $5 \%$ dextrose, administered 3 doses of intravenous (IV) neostigmine + atropine, and transferred to the intensive care unit (ICU). 

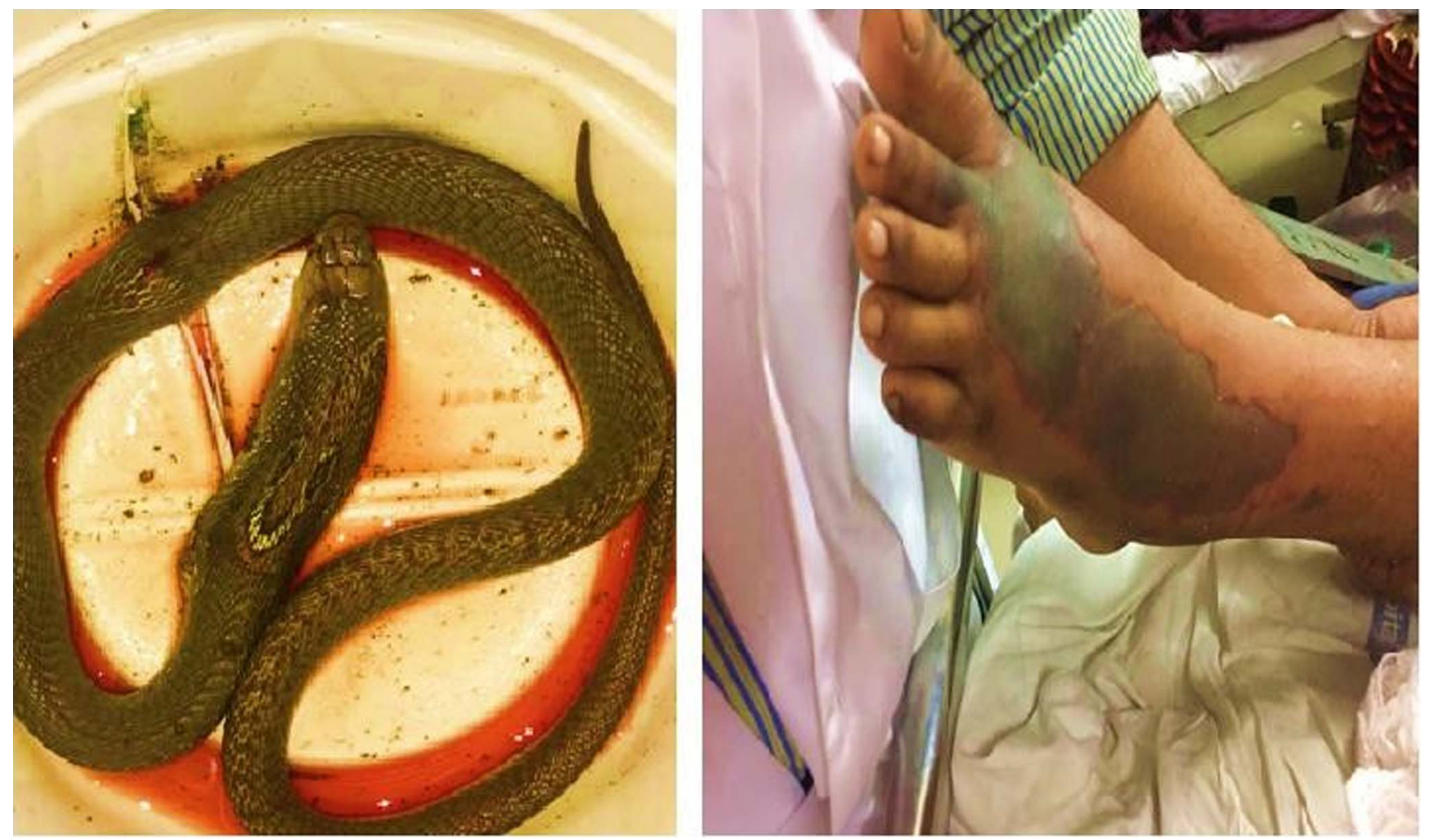

Figure. (Left) The Indian spectacled cobra that bit the patient. (Right) Cellulitis developed at the bite site by day 7.

On day 2, a repeat dose of snake antivenom was administered, and the patient showed signs of improvement. On day 3 , the patient was conscious and oriented, and she was extubated. Postextubation, the patient maintained $\mathrm{SpO}_{2}$ of $96 \%$ on ambient air. Because of the progressive improvement in the patient's condition, she was transferred to the wards on day 4 . However, at the site of the bite, a reddish hue progressively developed, in addition to swelling and an increase in local temperature. On day 6 , the patient suddenly developed severely blurred and diminished vision associated with ptosis and slurred speech. Full neurologic examination found no neurologic deficit other than the visual findings: the patient had bilateral ptosis and only perceived light in both eyes. Computed tomography scan and magnetic resonance imaging were normal. After consultation with the ophthalmologist and neurologist, delayed neurotoxicity from the venom was suspected, and an additional dose of snake antivenom was administered. Visual evoked potential indicated bilateral axonopathic optic neuropathy with maculopathy indicative of optic neuritis. The patient was started on IV methylprednisolone $500 \mathrm{mg}$ daily for 7 days and then transitioned to oral prednisone $40 \mathrm{mg} 3$ times daily that was tapered by $20 \mathrm{mg}$ every 7 days. By day 7 , the swelling had become tender, indicative of cellulitis (Figure, right panel). IV antibiotics amoxicillin clavulanate $1.2 \mathrm{~g}$ twice daily and metronidazole $500 \mathrm{mg} 3$ times daily were administered, the limb was elevated, and a magnesium sulphate dressing was applied.

The patient showed gradual improvement in her symptoms by day 4 of IV methylprednisolone and was discharged 14 days after initial presentation. After discharge, the patient was followed monthly for 3 months. She fully recovered her visual acuity within 1 month. She showed no residual effects and resumed her job and daily activities.

\section{DISCUSSION}

Our patient initially appeared to be a straightforward, classic case of neurotoxic snakebite without an accompanying diagnostic dilemma. The patient was treated per WHO guidelines ${ }^{5}$ and rapidly recovered from her initial symptoms. What was baffling was her deterioration on day 6 and the development of optic neuropathy. We found only 7 case reports of optic neuritis following snakebite. ${ }^{6-12}$ All 7 patients had received snake antivenom. As with our patient, the deterioration of vision occurred on day 6 in 3 of the reported cases. ${ }^{7-9}$

Hypotheses for the cause of optic neuritis after snakebite are the snake venom itself, ${ }^{11}$ extensive hemorrhages, allergy to snake antivenom, ${ }^{9}$ and capillary damage. ${ }^{8}$ Indirect evidence supports each theory. For example, Mathur published a case of optic neuritis following the administration of snake antivenom after a nonpoisonous snakebite and claimed that the antivenom led to the optic neuritis. ${ }^{9}$ Rao reported the sudden development of blindness following a cobra bite and attributed it to the toxic effects of the venom. ${ }^{11}$

In our case, we were unable to ascertain if a reaction to the snake antivenom or the direct toxic effect of the snake venom was the cause of the patient's optic neuritis. Irrespective of the etiology of optic neuritis, the treatment is the same: steroids. Steroids were used to treat optic neuritis following snakebite in 5 of the 7 reported cases. The patients' 
vision was reported to improve in all cases, but improvement took longer for the 2 patients who did not receive steroids.

Neuropathy is a known delayed complication of envenomation. ${ }^{13}$ Neuropathy is often first noticed after recovery from the acute phase of ventilation and ICU care. In a study of 210 patients, 38 patients who were bitten by the common krait developed delayed neurologic deficits. ${ }^{13}$ Subsequently, 14 of them also developed nerve conduction defects that persisted from 2 weeks to 6 months, followed by complete recovery. After the acute phase of envenoming, polyneuropathy has been observed for several months in snake-bitten patients. ${ }^{14}$ Cases of Guillain-Barré syndrome have also been reported. ${ }^{15}$

Further study and evaluation are needed to identify the factors responsible for long-term neurologic effects. Possible explanations include delayed immune-mediated reactions to toxins or antivenom and ongoing axonal damage caused by neurotoxins.

\section{CONCLUSION}

Snake antivenom is the mainstay of treatment for venomous snakebite, but clinicians should keep in mind the possible reactions and complications, both immediate and delayed. The treating physician should not become complacent once the patient starts to improve but should be alert to delayed manifestations. Optic neuritis following snakebite is rare but does occur. The prognosis is generally good, particularly if steroids are administered.

\section{ACKNOWLEDGMENTS}

The authors have no financial or proprietary interest in the subject matter of this article. The authors wish to thank Medkrux Research Group and the patient.

\section{REFERENCES}

1. Gutiérrez JM, Calvete JJ, Habib AG, Harrison RA, Williams DJ, Warrell DA, eds. Snakebite envenoming. Nature Reviews Disease Primers. World Health Organization. www.who.int/snakebites/resources/nrdp-2017-63/en/. Published September 2017. Accessed May 1, 2019.
2. Kasturiratne A, Wickremasinghe AR, de Silva N, et al. The global burden of snakebite: a literature analysis and modelling based on regional estimates of envenoming and deaths. PLoS Med. 2008 Nov 4;5(11):e218. doi: 10.1371/journal.pmed.0050218.

3. Mohapatra B, Warrell DA, Suraweera W, et al. Snakebite mortality in India: a nationally representative mortality survey. PLoS Negl Trop Dis. 2011 Apr 12;5(4):e1018. doi: 10.1371/journal.pntd.0001018.

4. Mukherjee AK. Green medicine as a harmonizing tool to antivenom therapy for the clinical management of snakebite: the road ahead. Indian J Med Res. 2012 Jul;136(1):10-12.

5. Warrell DA, ed. Guidelines for the management of snakebites. 2nd ed. World Health Organization Regional Office for South-East Asia. www.who.int/snakebites/resources/9789290225300/en/. Published August 2016. Accessed July 8, 2020.

6. Chopra PC. Optic neuritis after cobra bite. Indian Med Gaz. 1939;74:420.

7. Davenport RC, Budden FH. Loss of sight following snake bite. Br J Ophthalmol. 1953 Feb;37(2):119-121. doi: 10.1136/bjo.37.2.119.

8. Guttmann-Friedmann A. Blindness after snake-bite. $\mathrm{Br} J$ Ophthalmol. 1956 Jan; 40(1):57-59. doi: 10.1136/bjo.40.1.57.

9. Mathur SP. Allergy to antivenine serum. Br J Ophthalmol. 1959 Jan;43(1):50-51. doi: 10.1136/bjo.43.1.50.

10. Sahai AS, Sinha RH. Bilateral blindness following snake bite. Indian J Ophthalmol. 1978 Oct;26(3):16.

11. Rao KV. Optic neuritis and ophthalmoplegia caused by snake bite. Indian J Ophthalmol. 1981 Oct;29(3):243-245.

12. Sanghavi NG, Amin SK, Naik RS. Bilateral optic neuritis following snake bite-a case report. J Assoc Physicians India. 1982 Feb;30(2):117-118.

13. Kularatne SAM. Common krait (Bungarus caeruleus) bite in Anuradhapura, Sri Lanka: a prospective clinical study, 1996-98. Postgrad Med J. 2002 May;78(919):276-280. doi: $10.1136 /$ pmj.78.919.276.

14. Seneviratne $U$, Dissanayake $S$. Neurological manifestations of snake bite in Sri Lanka. J Postgrad Med. 2002 Oct-Dec;48(4):275-278; discussion 278-279.

15. Ranawaka UK, Lalloo DG, de Silva HJ. Neurotoxicity in snakebite-the limits of our knowledge. PLoS Negl Trop Dis. 2013 Oct 10;7(10):e2302. doi: 10.1371/journal.pntd.0002302.

This article meets the Accreditation Council for Graduate Medical Education and the American Board of Medical Specialties Maintenance of Certification competencies for Patient Care and Medical Knowledge.

(C2021 by the author(s); licensee Ochsner Journal, Ochsner Clinic Foundation, New Orleans, LA. This article is an open (C) access article distributed under the terms and conditions of the Creative Commons Attribution (CC BY) license (creativecommons.org/licenses/by/4.0/legalcode) that permits unrestricted use, distribution, and reproduction in any medium, provided the original author(s) and source are credited. 